\title{
Efectos de una intervención multidisciplinaria en pacientes con hipertensión arterial primaria
}

\section{Multidisciplinary intervention effects in patients with primary arterial hypertension}

\author{
Raquel García Flores, Christian Oswaldo Acosta Quiroz, \\ Yessica Mercedes León Ibarra, Brianda Militza Lagarda Vega, \\ Claudia García Hernández y Teresa Iveth Sotelo Quiñonez ${ }^{1}$
}

\begin{abstract}
RESUMEN
Las intervenciones multidisciplinarias han demostrado ser efectivas para mejorar la adherencia al tratamiento y la calidad de vida en pacientes con hipertensión (HTA). Sin embargo, son pocos los estudios que incluyen al psicólogo como parte del equipo médico, por lo que se evaluaron los efectos de una intervención multidisciplinaria en pacientes con HTA. Participaron trece mujeres con HTA y se aplicaron los inventarios de Calidad de Vida y Salud, de Ansiedad de Beck y el Multicultural de la Expresión de la Cólera y la Hostilidad en el pretest, el postest y el seguimiento. Las pacientes participaron en una intervención grupal de 16 sesiones en las que se incluyeron a un médico internista, una enfermera, una nutrióloga, un licenciado en ciencias del ejercicio físico y dos psicólogas. Las técnicas aplicadas fueron las de exposición de información, control de estímulos, reestructuración cognitiva, modelo de solución de problemas, reforzamiento positivo y modelamiento. Mediante la prueba de rangos de Wilcoxon se obtuvieron diferencias estadísticamente significativas en ansiedad entre el pretest y el seguimiento. Respecto a la calidad de vida y enojo, en algunas áreas se identificó una disminución estadísticamente significativa: aislamiento, relación con el médico, síntomas físicos y enojo estado. Respecto a la significancia clínica, ocho pacientes disminuyeron su sintomatología ansiosa, nueve mejoraron la calidad de vida total y cinco mejoraron clínicamente en las subescalas de enojo-rasgo, enojo externo y control del enojo externo. Se recomienda realizar más estudios en población masculina y en el ámbito laboral para facilitar la asistencia a las sesiones.
\end{abstract}

Palabras clave: Hipertensión; Calidad de vida; Intervención cognitivo-conductual; Enojo; Ansiedad.

\begin{abstract}
Multidisciplinary interventions have shown effectiveness in improving adherence to treatment and quality of life in patients with hypertension. However, few studies include psychologists as part of the medical team. The present study examined the effects of a multidisciplinary intervention in 13 female patients with hypertension. Measurement included the Quality of Life and Health Inventory, the Beck Anxiety Inventory and the Latin-American Multicultural State-Trait Anger Expression scale. Participants were exposed to a 16-sessions group intervention. The health caregiving team included one medical internist, one nurse, one nutritionist, one physical exercise expert and two psychologists. Intervention techniques included information exposure, stimulus control, cognitive restructuring, problem solving, positive reinforcement and modeling. The Wilcoxon rank test
\end{abstract}

\footnotetext{
${ }^{1}$ Departamento de Psicología, Instituto Tecnológico de Sonora, 5 de febrero 818 Sur, Col. Centro, 85000 Ciudad Obregón, Son., México, tel. (644)410-09-00, ext. 2457, correos electrónicos: raquel.garcia@itson.edu.mx, christian.oswaldo@itson.edu.mx, yessica_leon27@hotmail.com, militza_lag@hotmail.com, cgarcia@itson.edu.mx y teresa.sotelo@itson.edu.mx. Artículo recibido el 3 de marzo y aceptado el 16 de mayo de 2017.
} 
showed statistically significant differences in anxiety between pretest and follow-up. Some quality of life areas such as isolation, relationship with the physician, physical symptoms and anger status showed statistically significant improvement. Regarding clinical significance, eight patients decreased their anxiety, nine improved their total quality of life, and five patients improved clinically in trait-anger, external anger and external anger control subscales. It is recommended to carry out further studies in male populations at the work place in order to facilitate attendance to the sessions.

Key words: Hypertension; Quality of life; Cognitive behavioral intervention; Anger; Anxiety.

\section{INTRODUCCIÓN}

$\mathrm{L}$ a hipertensión arterial (HTA) es un síndrome de etiología múltiple caracterizado por la elevación persistente de las cifras de presión arterial en cifras iguales o mayores a 140/90 $\mathrm{ml} / \mathrm{Hg}$ (Organización Mundial de la Salud [OMS], 2015). Es uno de los problemas crónicos de salud que afecta gravemente a la población mexicana (Instituto Nacional de Estadística y Geografía [INEGI], 2016). En la Encuesta Nacional de Salud y Nutrición (ENSANUT), llevada a cabo en 2012 por el Instituto Nacional de Salud Pública (INSP), pudo identificarse que la prevalencia de HTA en México fue de $31.5 \%$ (uno de cada tres personas), de las cuales $47.3 \%$ desconocía padecer esta enfermedad. El grupo de edad de mayores de 60 años tuvo una prevalencia 3.4 veces más alta de HTA que el grupo de edad más joven (20-29 años). La prevalencia de HTA es más alta en la región norte del país (36.4\%) que en el sur $(28.5 \%)$, en las localidades urbanas $(31.9 \%)$ que en las rurales $(29.9 \%)$ y en el nivel socioeconómico alto (31.1\%) comparado con el bajo (29.7\%). El estado de Sonora ocupa el quinto lugar en relación a la prevalencia de este padecimiento, con $38 \%$, siete puntos porcentuales por arriba de la media nacional.

De los adultos con HTA diagnosticada por un médico, solo $73.6 \%$ reciben tratamiento farmacológico y menos de la mitad tiene un control adecuado de la misma, lo que es preocupante ya que es uno de los factores de riesgo para padecer enfermedad cardiovascular y cerebrovascular y falla renal, que son causas importantes de mortalidad en el país (ISPN, 2012). Por ejemplo, las enfermedades del corazón ocupan el primer lugar como causa de muerte en México; en 2015 fallecieron 128,731 mexicanos a causa de este tipo de padecimientos (INEGI, 2016). En Estados Unidos, es la población latina (mayoritariamente de descendencia mexicana) la que presenta los porcentajes más bajos en el control de la HTA (Schoenthaler et al., 2015).

El tratamiento de la HTA es complejo en virtud de que requiere cambios permanentes en el estilo de vida de los pacientes, tales como evitar el consumo de tabaco y alcohol, una dieta baja en grasas saturadas y sodio, un elevado consumo de verduras y granos de cereales enteros, actividad física moderada un mínimo 150 minutos a la semana, toma de medicamentos y monitoreo constante de la presión arterial (Campos et al., 2013).

La adherencia al tratamiento consiste en el grado en el cual los pacientes siguen las recomendaciones prescritas por el médico o el profesional de la salud (Vermeire, Hearnshaw, Van Royen y Denekens, 2001). En el manejo de las enfermedades crónicas, cerca de la mitad de los pacientes no se adhieren a la toma de medicamentos, citas médicas, actividad física y cambios en la alimentación (DiMatteo, Haskard-Zolnierek y Martin, 2012). Por ejemplo, Doubova, Martínez, Aguirre y Pérez (2017) identificaron en un estudio que $16 \%$ de los pacientes no recibió o no entendió las dosis de sus medicamentos, 93\% desconocía los efectos adversos de los medicamentos que tomaba, 59\% no recibió o no entendió las razones para realizar actividad física y $30 \%$ no recibió o no entendió el tipo de ejercicio ni su duración y frecuencia semanal. En cuanto a las conductas, $46 \%$ de los pacientes presentó una baja adherencia farmacológica, $47 \%$ no llevaba a cabo una actividad física regular, $31 \%$ consumía pocas frutas y verduras y $54 \%$ consumía frecuentemente comidas con un alto contenido de sal. Las personas con menor nivel educativo eran quienes menos información tenían acerca de los medicamentos y sus efectos.

En América Latina también se han desarrollado estudios en relación a la adherencia en pacientes con hipertensión. En un estudio llevado a cabo en Colombia con pacientes con HTA se pudo determinar que solo 9.4\% mostraba adherencia no 
farmacológica (estilo de vida saludable), 75\% no realizaba actividad física regular y más de 19\% de la población consumía sal frecuentemente, lo que indica que los cambios en el estilo de vida son difíciles de ponerse en práctica (Rodríguez et al., 2015).

Los factores que pueden influir en la escasa adherencia son la falta de información sobre la enfermedad y el tratamiento, las creencias inapropiadas sobre los beneficios y la eficacia del tratamiento prescrito, las barreras reales o percibidas de los tratamientos prescritos, las demandas excesivas de los mismos y la ausencia de apoyo social (Haskard-Zolnierek y DiMatteo, 2009).

En relación con lo anterior, Ávila, Gómez, Yam, Vega y Franco (2013) llevaron a cabo un estudio en Guanajuato (México) para identificar las barreras percibidas por pacientes con HTA, las cuales fueron las siguientes: creencias inapropiadas respecto al tratamiento, falta de conocimientos y habilidades para la dieta, descontento con la dieta, falta de apoyo familiar, dificultades económicas, falta de conocimientos sobre la medicación, indiferencia hacia el ejercicio, y desconfianza y temor a los estudios de laboratorio. También las creencias del paciente sobre los síntomas de la HTA se relacionan con la adherencia al tratamiento; por ejemplo, en un estudio realizado por Granados, Roales, Moreno e Ybarra (2007) se encontró que 83\% de pacientes con HTA no seguían el tratamiento debido a que creían que su presión arterial "estaba bien" sin tener datos objetivos sobre la misma, y $70 \%$ retomaba el tratamiento solamente cuando se manifestaban los síntomas (dolor de cabeza, taquicardia, etc.).

Si se considera que en muchos casos la HTA es asintomática, entonces un alto porcentaje de pacientes no sigue su tratamiento de manera adecuada. De acuerdo con Jankowska-Polańska, Uchmanowicz, Dudek y Mazur (2016), se ha demostrado que entre más información sobre la hipertensión tiene una persona, mejor se adherirá al tratamiento.

Un elemento importante a considerar es la calidad de vida relacionada con la salud, misma que se define como el grado de funcionamiento de una persona en su vida y su percepción de bienestar en relación a su salud en las áreas física, mental y social (Hays y Reeve, 2017). Se han llevado a cabo estudios comparativos sobre la calidad de vida en poblaciones con y sin HTA, mismos que han permitido afirmar que dicha calidad es menor en personas que la sufren, principalmente cuando está asociada a trastornos emocionales (Trevisol, Moreira, Kerkhoff, Fuchs y Fuchs, 2011; Xianglong et al., 2016).

La ansiedad y el enojo son estados emocionales que influyen en la presión arterial y la frecuencia cardiaca debido a que activan el sistema simpático-suprarrenal-medular y el eje hipotálamo-hipófisis-adrenal-cortical, seguidos de un aumento de los niveles séricos de catecolaminas y cortisol (Kubzansky y Kawachi, 2000). Se ha identificado que las personas que padecen HTA manifiestan niveles más elevados de ansiedad y experimentan con mayor frecuencia pensamientos hostiles y agresivos (Magan, Sanz, Espinosa y García, 2013). Doubova et al. (2017) señalan que la comorbilidad más frecuente en pacientes con hipertensión fue la ansiedad (23\%). De la misma forma, Maatouk et al. (2016), en un estudio con pacientes adultos mayores con hipertensión, identificaron que $14 \%$ de ellos sufría trastorno de ansiedad generalizada.

En relación al enojo, un estudio realizado por Hosseini, Mokhberi, Mohammadpour, Mehrabianfard y Lashak (2011) en pacientes con HTA pudo identificar que el enojo-rasgo y la supresión del enojo eran más elevados en personas con HTA que sin ella. De igual manera, compararon el enojo y el estrés en personas con y sin hipertensión, hallando que los primeros mostraron 9.7 veces más estrés y 19 veces más enojo interno que las segundas.

Se ha considerado la intervención multidisciplinaria como una buena opción para mejorar la adherencia al tratamiento y la calidad de vida en pacientes con HTA. En un estudio de Houle, Chatterley y Tsuyuki, (2014) se considera la intervención conjunta de médicos, enfermeras, dietistas y fisioterapeutas; sin embargo, son pocos los estudios en los que se incorpora al psicólogo como miembro del equipo multidisciplinario.

En el área psicológica, las técnicas cognitivo-conductuales han mostrado ser efectivas para mejorar la adherencia al tratamiento y la calidad de vida en pacientes con HTA (Annesi, 2013; Riveros, Ceballos, Laguna y Sánchez-Sosa, 2005). Tales técnicas se basan en la psicología del aprendizaje y el objetivo de la intervención es el cam- 
bio conductual, cognitivo y emocional al modificar o eliminar la conducta desadaptada y enseñar conductas adaptativas (Ruiz, Díaz y Villalobos, 2012). Las técnicas cognitivo-conductuales que han demostrado mayor efectividad para facilitar la adherencia al tratamiento y el manejo emocional en pacientes con hipertensión son, a saber: el control de estímulos (toma de medicamentos, alimentación), la psicoeducación, la reestructuración cognitiva, la respiración diafragmática, el modelamiento (sobre todo en la toma de la presión arterial), el reforzamiento positivo y el modelo de solución de problemas de D'Zurilla (cf. Asghari, Faramarzi y Mohammmadi, 2016; Riveros, Ceba1los et al., 2005; Riveros, Cortazar et al., 2005; Vocks, Ockenfels, Jürgensen, Mussgay y Rüddel, 2004). López, Rodríguez, Vázquez y Alcázar (2012) han demostrado asimismo que las técnicas cognitivo-conductuales son útiles para disminuir el enojo.

Respecto a la adherencia, Hinderliter et al. (2014) evaluaron el impacto de tres tipos de intervenciones durante cuatro meses: una tradicional, otra de recomendaciones dietéticas y una más que incluía estrategias cognitivo-conductuales para llevar a cabo los cambios de conducta. Las variables evaluadas fueron la presión arterial, el peso corporal, el contenido de la dieta, el ejercicio, los medicamentos antihipertensivos y los eventos cardiovasculares. Se observó que la intervención con recomendaciones sobre la alimentación y la que incluía una intervención cognitivo-conductual fueron efectivas tras ocho meses de seguimiento. Sin embargo, no se evaluaron las variables psicológicas, las cuales, como se mencionó anteriormente, influyen de manera importante en el control del padecimiento.

Por lo tanto, el objetivo del presente estudio fue analizar los efectos de una intervención multidisciplinaria en los niveles de presión arterial, actividad física, calidad de vida, ansiedad y enojo en personas con hipertensión arterial.

\section{MÉTODO}

\section{Participantes}

En este estudio participaron trece pacientes hipertensas jubiladas, con edades de entre 55 y 70 años y media de 60 . Todas las participantes eran casa- das, con una escolaridad de secundaria y con más de cinco años desde el diagnóstico de la enfermedad. Las pacientes no presentaban complicaciones crónicas derivadas del padecimiento.

\section{Instrumentos}

Carta de Consentimiento Informado, en la cual se informaban las características de la intervención multidisciplinaria.

Entrevista semiesctructurada para pacientes con hipertensión (Fernández, 2009). Mediante la misma, se recabaron los datos generales y las conductas de adherencia al tratamiento. Se exploraron los problemas relacionados con la toma de medicamentos, la frecuencia del automonitoreo de la presión arterial, el consumo de alimentos altos en sodio y grasas saturadas y la frecuencia de actividad física.

Inventario de Calidad de Vida y Salud (INCAVISA) (Riveros, Sánchez y Del Águila, 2009). Es un cuestionario autoaplicable cuya administración varía entre 15 y 20 minutos. Consta de 68 reactivos con tipo de respuesta Likert: "nunca" $(0 \%)$, "casi nunca" (20\%), "pocas veces" (40\%), "frecuentemente" (60\%), "casi siempre" (80\%) y "siempre" (100\%). Para medir la calidad de vida de pacientes, evalúa doce áreas: preocupación, desempeño físico, aislamiento, percepción corporal, funciones cognitivas, actitud ante el tratamiento, tiempo libre, vida cotidiana, familia, redes sociales, dependencia médica y relación con el médico. Cada área consta de cuatro reactivos. Con el objetivo de calcular la consistencia interna del inventario, se computaron los coeficientes alfa de Cronbach, los cuales variaron de .93 en el área de vida cotidiana a .68 en el de actitud ante el tratamiento.

Inventario de Ansiedad de Beck. En la versión mexicana de Robles, Varela, Jurado y Páez (2001), permite evaluar síntomas de ansiedad. El tiempo de aplicación varía de 5 a 10 minutos. Las respuestas se clasifican en "poco o nada", "más o menos", "moderadamente" y "severamente. El instrumento muestra una consistencia interna de .83 y una validez de constructo que arrojó cuatro factores principales congruentes con los referidos por los autores de la versión original: subjetivo, neurofisiológico, autonómico y pánico. 
Inventario Multicultural de la Expresión de la Cólera y la Hostilidad (ML-STAXI) (Moscoso y Spielberger, 1999). Puede aplicarse de forma individual o colectiva. La confiabilidad medida con el coeficiente alfa de Cronbach va de .65 a .86 . El instrumento mide el Enojo-estado (diez reactivos), que se refiere al enojo que sentía la persona al momento de contestar el inventario y que se divide en dos subescalas: "Sintiendo enojo" (cinco reactivos) y "Deseo de expresar el enojo física o verbalmente" (cinco reactivos); el enojo-rasgo (diez reactivos), que evalúa la disposición o vulnerabilidad para reaccionar generalmente con enojo y que tiene dos subescalas: "Temperamento de enojo" (cinco ítems) y "Reacción de enojo" (cinco ítems); expresión y control del enojo, que se divide en dos subescalas: "Expresión externa" (cinco reactivos), que se refiere a mostrar el enojo hacia otras personas, y "Expresión interna" (seis reactivos), que indica que el individuo suprime su enojo, se aleja de los demás y guarda rencores; control del enojo, con dos subescalas: "Control externo" (siete reactivos), que mide el grado en que la persona intenta dominar o controlar su propia conducta cuando está enojado, y "Control interno" (seis reactivos), que evalúa los intentos por calmarse o relajarse para disminuir el enojo.

\section{Procedimiento}

Se hizo la invitación a los pacientes en la sala de espera de un hospital público de Ciudad Obregón, Son. (México). Una vez que las pacientes acepta- ron participar, firmaron la carta de consentimiento informado y se les aplicaron los instrumentos mencionados de manera individual una semana antes de la intervención.

El equipo de trabajo estuvo conformado por un médico internista, una enfermera, una nutrióloga, un licenciado en ciencias del ejercicio físico (LCEF), todos ellos empleados de la unidad de salud, y dos psicólogas (becarias tesistas) debidamente entrenadas por una psicóloga con doctorado y experiencia en terapia cognitivo-conductual. Dicho equipo sostuvo dieciséis sesiones grupales, con una frecuencia de dos veces a la semana y una duración de una hora y media cada una. Sólo hubo un grupo de intervención y la intervención se llevó a cabo a lo largo de dos meses en total.

Las cinco primeras sesiones se realizaron en la unidad de salud, y las subsecuentes en un centro comunitario en las que los pacientes participaron en sesiones de activación física guiadas por el LCEF. Después de la sesión de actividad física de 45 minutos, las psicólogas impartían el tema correspondiente en el centro comunitario. El seguimiento se llevó a cabo desde el momento en que concluyó la intervención. Las técnicas aplicadas fueron las siguientes: exposición de información, control de estímulos, ensayo conductual, reestructuración cognitiva, modelo de solución de problemas, reforzamiento positivo, modelamiento (Ruiz et al., 2012). En la Tabla 1 se describen los temas impartidos, el contenido en cada sesión y las técnicas implementadas.

Tabla 1. Descripción de la intervención multidisciplinaria.

\begin{tabular}{|l|l|l|}
\hline \multicolumn{1}{|c|}{ Sesión } & \multicolumn{1}{|c|}{ Tema } & \multicolumn{1}{c|}{ Contenido/Técnicas } \\
\hline 1 & Integración grupal & $\begin{array}{l}\text { Presentación del objetivo, establecimiento de reglas e identificación de parti- } \\
\text { cipantes (Exposición). }\end{array}$ \\
\hline 2 & $\begin{array}{l}\text { Definición de hipertensión } \\
\text { y factores relacionados }\end{array}$ & $\begin{array}{l}\text { Descripción de la fisiología y tratamiento médico de la HTA por parte del mé- } \\
\text { dico internista. Efectos de las emociones y conductas en la presión arterial por } \\
\text { parte de las psicólogas (Exposición). }\end{array}$ \\
\hline 3 & $\begin{array}{l}\text { Automonitoreo de la presión } \\
\text { arterial }\end{array}$ & $\begin{array}{l}\text { Demostración por una enfermera, apoyada por las psicólogas, de los pasos } \\
\text { para medir la presión arterial (Modelamiento y ensayos conductuales). }\end{array}$ \\
\hline 4 & Alimentación saludable & $\begin{array}{l}\text { Información impartida por una nutrióloga sobre los alimentos y sus porciones } \\
\text { recomendables para personas con HTA. Las psicólogas describieron procedi- } \\
\text { mientos para facilitar el cambio, como el control de estímulos (Exposición y } \\
\text { control de estímulos). }\end{array}$ \\
\hline 5 & Actividad física & $\begin{array}{l}\text { Descripción por el LCEF de las condiciones para realizar la actividad física en } \\
\text { pacientes con HTA (Exposición). }\end{array}$ \\
\hline
\end{tabular}




\begin{tabular}{|l|l|l|}
\hline $16,7,8,9$ & Manejo emocional & $\begin{array}{l}\text { Descripción de la relación entre pensamiento, emoción y conducta. Por medio } \\
\text { de registros, se pidió a las participantes que identificaran pensamientos asocia- } \\
\text { dos a las emociones que experimentaban cotidianamente, sobre todo durante } \\
\text { episodios de ansiedad y enojo. Se generaron pensamientos alternativos más } \\
\text { funcionales, para que los pacientes identificaran el cambio en la intensidad de } \\
\text { la emoción o la presencia de emociones más adaptativas. Se practicó la técnica } \\
\text { de respiración diafragmática al finalizar cada sesión (Modelamiento, reestruc- } \\
\text { turación cognitiva, ensayo conductual). }\end{array}$ \\
\hline 10 & Identificación de fortalezas & $\begin{array}{l}\text { Verbalización por parte de las participantes de las cualidades que considera- } \\
\text { ban poseer y reconocimiento de las fortalezas de las demás (Reforzamiento } \\
\text { positivo). }\end{array}$ \\
\hline $11,12,13$ & Modelo de solución de problemas & $\begin{array}{l}\text { Descripción de la técnica, solicitándoles a las participantes que la pusieran en } \\
\text { práctica en casos hipotéticos. Después, aplicación en parejas de la técnica en } \\
\text { problemas propios (Modelamiento). }\end{array}$ \\
\hline 14 & $\begin{array}{l}\text { Fortaleciendo la alimentación } \\
\text { saludable }\end{array}$ & $\begin{array}{l}\text { Recomendaciones por parte de la nutrióloga para que las participantes conti- } \\
\text { nuaran con el cambio de conducta alimentaria (Exposición). }\end{array}$ \\
\hline 15 & Perdonando errores & $\begin{array}{l}\text { Identificación por parte de las participantes de situaciones específicas que se } \\
\text { relacionaban con episodios de enojo y pensamientos detonantes, y generación } \\
\text { de pensamientos alternativos que disminuían la reacción emocional (Reestruc- } \\
\text { turación cognitiva). }\end{array}$ \\
\hline 16 & $\begin{array}{l}\text { Manifestación por las participantes de los cambios logrados tras el taller, for- } \\
\text { taleciendo en ellas el compromiso de mantener el comportamiento saludable. } \\
\text { Cada participante preparó un platillo saludable para compartir con los demás } \\
\text { (Reforzamiento positivo). }\end{array}$ \\
\hline
\end{tabular}

Cada quince días el LCEF medía la presión arterial de las participantes al finalizar la sesión, por lo que se hicieron ocho mediciones en total. Se aplicaron nuevamente los instrumentos una semana después de haber finalizado la intervención y se llevó a cabo un seguimiento a un mes.

\section{RESULTADOS}

En la entrevista semiestructurada llevada a cabo antes de la intervención, cuatro pacientes dijeron tener problemas para controlar su presión arterial; la mitad de ellas señaló llevar a cabo actividades físicas, pero de manera esporádica y con ciertas dificultades; seis reportaron no tener control sobre los alimentos altos en sodio; cuatro reportaron una alimentación baja en calorías y grasa; todas dijeron tomar café diariamente, y la mayoría tuvo problemas para tomar los medicamentos (olvidos, horarios diferentes al recomendado y dosis inadecuadas).

Al finalizar la intervención, todas las participantes reportaron en la entrevista tomar los medicamentos tal como habían sido prescritos por el médico; asistían diariamente a la sesión de actividad física con el LCEF de la unidad de salud, y también comunicaron que disminuyeron el consumo de alimentos altos en sodio y grasa. Respecto a la ingesta de café, señalaron tener dificultades para dejar de consumirlo ya que era un hábito muy arraigado. No contaron con autorregistros de su conducta, por lo que las mediciones de la presión arterial fueron tomadas como un indicador de la adherencia, además de lo reportado en las entrevistas.

Se calculó la significancia estadística de los cambios en los puntajes de calidad de vida, ansiedad y enojo entre el pretest y el postest, y entre el pretest y el seguimiento, por medio de la prueba de rangos asignados de Wilcoxon. Se eligió este estadístico por el tamaño del grupo, el nivel de medición ordinal, el diseño experimental utilizado y la comparación de dos muestras relacionadas en las que cada sujeto sirve como su propio control (Siegel y Castellan, 1995). Las diferencias pretest-seguimiento respecto a la ansiedad arrojó $p=.008$; además, la intensidad de los síntomas de ansiedad también disminuyó en cuanto a que si en el pretest ocho participantes manifestaron niveles de ansiedad moderada y severa, en el postest solo fueron seis y en el seguimiento únicamente cuatro, lo que indica que la frecuencia de pacientes con sintomatología ansiosa moderada y severa 
descendió en $50 \%$. Respecto a la calidad de vida y enojo, en algunas áreas se identificó una disminución estadísticamente significativa (aislamiento, relación con el médico, síntomas físicos y enojo estado), con probabilidades asociadas de entre $p=.001$ y $p=.027$.

Respecto a la significancia clínica, se realizó el cálculo del cambio clínico objetivo, que consiste en la diferencia entre el pretest y el postest dividida entre el pretest (Cardiel, 1994). Se considera que el cambio clínico objetivo es significativo cuando el resultado es mayor a 0.20 , ya sea positivo o negativo. Los cálculos se realizaron con los datos del pretest-postest y el pretest-seguimiento. En la Tabla 2 puede apreciarse el número de pacientes que mejoraron, empeoraron o permanecieron igual entre el pretest y el postest, y entre el pretest y el seguimiento, en el Inventario de Ansiedad de Beck y en cada una de las subescalas del INCAVISA. Como puede observarse, los niveles de ansiedad disminuyeron significativamente en ocho pacientes, lo que se muestra en negritas.

Tabla 2. Cambio clínico objetivo del Inventario de Ansiedad de Beck y el Inventario de Calidad de Vida y Salud.

\begin{tabular}{|l|c|c|c|c|c|c|}
\hline \multirow{2}{*}{\multicolumn{1}{|c|}{ Subescalas }} & \multicolumn{2}{|c|}{ Mejoró } & \multicolumn{2}{c|}{ Empeoró } & \multicolumn{2}{c|}{ Permaneció igual } \\
\cline { 2 - 7 } & $\begin{array}{c}\text { Pretest- } \\
\text { Postest }\end{array}$ & $\begin{array}{c}\text { Pretest- } \\
\text { Seguimiento }\end{array}$ & $\begin{array}{c}\text { Pretest- } \\
\text { Postest }\end{array}$ & $\begin{array}{c}\text { Pretest- } \\
\text { Seguimiento }\end{array}$ & $\begin{array}{c}\text { Pretest- } \\
\text { Postest }\end{array}$ & $\begin{array}{c}\text { Pretest- } \\
\text { Seguimiento }\end{array}$ \\
\hline Ansiedad & 8 & 8 & 2 & 1 & 3 & 4 \\
\hline Preocupación & 3 & 5 & 5 & 1 & 5 & 7 \\
\hline Aislamiento & 1 & 6 & 3 & 1 & 9 & 6 \\
\hline Desempeño físico & 1 & 5 & 3 & 3 & 9 & 5 \\
\hline Percepción corporal & 2 & 3 & 3 & 2 & 8 & 8 \\
\hline Funciones cognitivas & 5 & 5 & 4 & 3 & 4 & 5 \\
\hline Actitud ante el tratamiento & 3 & 5 & 3 & 1 & 7 & 7 \\
\hline Tiempo libre & 2 & 5 & 2 & 0 & 9 & 8 \\
\hline Vida cotidiana & 2 & 4 & 2 & 1 & 9 & 8 \\
\hline Familia & 1 & 3 & 2 & 1 & 10 & 9 \\
\hline Redes sociales & 3 & 6 & 4 & 1 & 6 & 6 \\
\hline Dependencia médica & 3 & 1 & 1 & 3 & 9 & 9 \\
\hline Relación con el médico & 3 & 9 & 4 & 1 & 6 & 3 \\
\hline Puntaje total INCAVISA & 4 & 9 & 2 & 1 & 7 & 3 \\
\hline
\end{tabular}

En relación a la calidad de vida, la mayor parte de las pacientes no mostró cambios importantes en cada subescala; sin embargo, destacó su mejoría en el seguimiento en varias subescalas, tales como preocupación, aislamiento, desempeño físico y redes sociales. En el puntaje total, nueve pacientes manifestaron una mejoría en la calidad de vida total. Llama la atención el hecho de que las pacientes mostraran una mejoría significativa tras el seguimiento a un mes de haber concluido la intervención.

En la Tabla 3 se muestra el número de pacientes que mejoraron, empeoraron y permanecieron igual del pretest al postest y del pretest al seguimiento en cada área del ML-STAXI. De manera similar a los resultados del Inventario de Calidad de Vida, la mayoría de las pacientes no mostró cambios clínicamente significativos; pese a ello, cinco pacientes lograron una importante mejoría en las subescalas de enojo-rasgo, enojo externo y control del enojo externo, y tres empeoraron en las subescalas de enojo interno y externo en el seguimiento.

Para las medidas de la presión arterial diastólica y sistólica se calculó un ANOVA para medidas repetidas debido a que el nivel de medición de la variable es intervalar. Se obtuvieron resultados estadísticamente significativos en la presión diastólica $(\mathrm{F}=3.35 ; p=.016)$. No se encontraron cambios significativos específicos de una sesión a otra, sino más bien de todas las sesiones en conjunto. 
Tabla 3. Cambio clínico objetivo del Inventario Multicultural de la Expresión de la Cólera y la Hostilidad.

\begin{tabular}{|l|c|c|c|c|c|c|}
\hline \multirow{2}{*}{ Escala } & \multicolumn{2}{|c|}{ Mejoró } & \multicolumn{2}{c|}{ Empeoró } & \multicolumn{2}{c|}{ Permaneció igual } \\
\cline { 2 - 7 } & $\begin{array}{c}\text { Pretest- } \\
\text { Postest }\end{array}$ & $\begin{array}{c}\text { Pretest- } \\
\text { Seguimiento }\end{array}$ & $\begin{array}{c}\text { Pretest- } \\
\text { Postest }\end{array}$ & $\begin{array}{c}\text { Pretest- } \\
\text { Seguimiento }\end{array}$ & $\begin{array}{c}\text { Pretest- } \\
\text { Postest }\end{array}$ & $\begin{array}{c}\text { Pretest- } \\
\text { Seguimiento }\end{array}$ \\
\hline Enojo-estado & 4 & 3 & 0 & 0 & 9 & 10 \\
\hline Enojo-rasgo & 4 & 5 & 2 & 3 & 7 & 5 \\
\hline Enojo interno & 2 & 2 & 1 & 4 & 10 & 7 \\
\hline Enojo externo & 2 & 5 & 2 & 3 & 9 & 5 \\
\hline Control del enojo interno & 2 & 2 & 1 & 4 & 10 & 7 \\
\hline Control del enojo externo & 2 & 5 & 2 & 3 & 9 & 5 \\
\hline
\end{tabular}

En la Figura 1 puede observarse el porcentaje acumulado de cada área del INCAVISA con el fin de evitar la pérdida o enmascaramiento de la varia- bilidad al utilizar promedios; cabe destacar que a mayor porcentaje, mayor deterioro.

Figura 1. Efectos de la intervención en el INCAVISA.

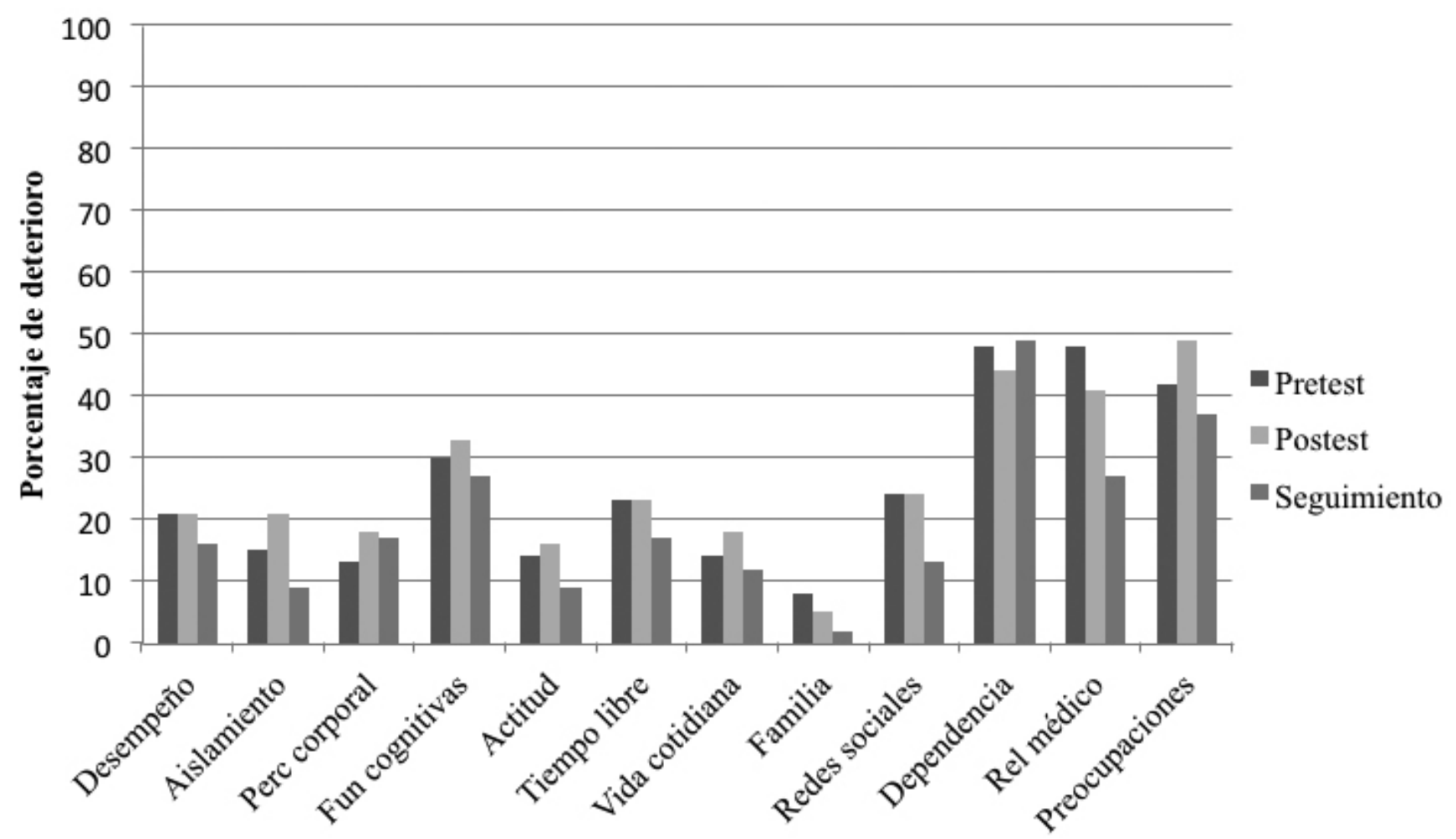

las áreas aun con diferente número de reactivos u opciones de respuesta en su composición (Riveros, Cortazar et al., 2005). Como puede observarse, la mayor parte de las pacientes se ubicaron en porcentajes bajos de deterioro antes de la intervención, lo que indica una alta funcionalidad, principalmente en el área Familia. Este hecho pudo influir para que varias pacientes no mostraran cambios en la intervención debido a que ya mostraban un alto grado de funcionalidad. Además, pudo detec- minución del deterioro en el postest, excepto en las variables Preocupaciones y Aislamiento.

Al finalizar la intervención, las pacientes reportaron una mejoría en su alimentación; procuraban medir las porciones y seleccionar alimentos bajos en sodio, disminuyeron asimismo el consumo de café, asistían diariamente a las sesiones de actividad física impartida por el LCEF del hospital y tomaban los medicamentos con mayor regularidad, según su reporte. 


\section{DISCUSIÓN}

La intervención multidisciplinaria demostró tener efectos benéficos en las participantes en algunos aspectos. Vale la pena mencionar que a pesar de que las pacientes mostraban un buen nivel de funcionamiento, pudieron mejorar aún más en algunos aspectos, lo cual les permitirá a futuro prevenir complicaciones crónicas derivadas del padecimiento. Pudo apreciarse que, tras la intervención, la preocupación y el aislamiento aumentaron, probablemente debido a que se percataron de la necesidad de cuidar su salud.

Uno de los datos más sobresalientes fue el hecho de que las participantes se involucraron en la actividad física diariamente, lo cual reforzó los cambios de las conductas saludables. Tal elemento es muy valioso ya que en la mayoría de los estudios únicamente se le pide al paciente que reporte la duración de la actividad física que realiza, sin realmente verificar si se llevó a cabo la misma (Dickman, Pintz, Gold y Kivlahan, 2012). Los beneficios de la actividad física se reflejaron en las mediciones de presión diastólica.

Por otra parte, la mayor parte de las participantes mostró cambios favorables, especialmente en aquellas áreas que tienen relación con aspectos sociales. Como pudo observarse, la sintomatología ansiosa disminuyó considerablemente, tal y como se ha demostrado en diversas investigaciones (Annesi, 2013; Riveros, Ceballos et al., 2005). Resultó de gran utilidad que diferentes especialistas se relacionaran con las pacientes en virtud de que podían resolver sus dudas, lo que quizá también influyó en el mayor puntaje obtenido en la subescala que evalúa la relación con el médico.
En las subescalas de enojo interno y control del enojo interno tres pacientes empeoraron, un efecto quizá debido a que pudieron identificar estos sentimientos con mayor sensibilidad al reforzarse la autoobservación al final de la intervención. Otro factor que pudo haber influido son los conflictos recientes que tal vez afectaron la evaluación en el seguimiento. Sería recomendable evaluar en futuros estudios las situaciones personales que podrían influir en los puntajes obtenidos en los instrumentos. Podrían añadirse sesiones de seguimiento presenciales o por vía telefónica para reforzar lo aprendido en la intervención (Hallberg, Ranerup y Kjellgren, 2016), así como incluirse sesiones para entrenar a los pacientes en comportamiento asertivo para mejorar su interacción social en situaciones que les generan molestia (López et al., 2012).

Se sugiere finalmente llevar a cabo más estudios de intervención multidisciplinaria que permitan reunir más evidencia de su impacto, especialmente en pacientes hombres, quienes participan menos en las intervenciones, y también sería importante poner en práctica programas de este tipo en el contexto laboral, pues para las personas que trabajan resulta complicado destinar un horario específico al cuidado de su salud.

El reducido grupo de pacientes es una de las limitaciones del estudio; de hecho, no hubo un grupo de comparación. Probablemente las pacientes que participaron ya se encontraban motivadas para cambiar sus conductas, por lo que se sugiere que en futuras investigaciones se amplíe el número de participantes y se contemple la posibilidad de tener un grupo control en lista de espera.

\section{REFERENCIAS}

Annesi, J. (2013). Association of multimodal treatment-induced improvements in stress, exercise volume, nutrition, and weight with improved blood pressure in severely obese women. International Journal of Behavioral Medicine, $20(3), 397-402$. doi: 10.1007/s12529-012-9240-7.

Asghari, E., Faramarzi, M. y Mohammmadi, A. (2016). The effect of cognitive behavioural therapy on anxiety, depression and stress in women with preeclampsia. Journal of Clinical and Diagnostic Research, 10(11), 4-7. doi: 10.7860/ $\mathrm{JCDR} / 2016 / 21245.8879$.

Ávila, G., Gómez A., P., Yam S., A., Vega A., G. y Franco C., B. (2013). Un enfoque cualitativo a las barreras de adherencia terapéutica en enfermos crónicos de Guanajuato, México. Aquichan, 13(3), 373-386.

Campos N., I., Hernández B., L., Rojas M., R., Pedroza, A., Medina G., C. y Barquera C., S. (2013). Hipertensión arterial: prevalencia, diagnóstico oportuno, control y tendencias en adultos mexicanos. Salud Pública de México, 55(Supl. 2), S144-S150. 
Cardiel H., M. (1994). ¿Cómo se evalúa la calidad de vida? En Asociación de Medicina Interna de México, A. C. (Ed.): Temas de medicina interna. Epidemiología clínica (pp. 359-368). México: Interamericana.

Dickman, K., Pintz, C., Gold, K. y Kivlahan, C. (2012). Behavior changes in patients with diabetes and hypertension after experiencing shared medical appointments. Journal of the American Academy of Nurse Practitioners, 24(1), 43-51. doi: 10.1111/j.1745-7599.2011.00660.x.

DiMatteo, M., Haskard-Zolnierek, K. y Martin, L. (2012). Improving patient adherence: a three-factor model to guide practice. Health Psychology, 6, 74-91. doi: 10.1080/17437199.2010.537592.

Doubova, S., Martinez V., I., Aguirre H., R. y Pérez C., R. (2017). Association of hypertension-related distress with lack of self-care among hypertensive patients. Psychology, Health \& Medicine, 22(1), 51-64. doi: 10.1080/13548506.2016.1239830.

Fernández, V. (2009). Eficacia de una intervención cognitivo-conductual en el tratamiento de la hipertensión arterial. Tesis inédita de doctorado. Madrid Universidad Complutense de Madrid.

Granados, G., Roales N., J. Moreno, E. e Ybarra, J. (2007). Creencias en síntomas y adherencia al tratamiento farmacológico en pacientes con hipertensión. International Journal of Clinical and Health Psychology, 7(3), 697-707.

Hallberg, A., Ranerup, I. y Kjellgren, K. (2016). Supporting the self-management of hypertension: Patients' experiences of using a mobile phone-based system. Journal of Human Hypertension, 30, 141-146.

Haskard-Zolnierek, K. y DiMatteo, M. (2009). Physician communication and patient adherence to treatment: A meta-analysis. Medical Care, 47, 826-834.

Hays, R. y Reeve, B. (2017). Measurement and modeling of health-related quality of life. International Encyclopedia of Public Health (pp. 241-252). Atlanta, GA: Elsevier, Inc.

Hinderliter, A., Sherwood A., Craighead, L., Lin, P., Watkins, L., Babyak, M. y Blumenthal, J. (2014). The long-term effects of lifestyle change on blood pressure: One-year follow-up of the ENCORE study. American Journal of Hypertension, 27(5), 734-41. doi: 10.1093/ajh/hpt183.

Hosseini, S., Mokhberi, V., Mohammadpour, R., Mehrabianfard, M. y Lashak, N. (2011). Anger expression and suppression among patients with essential hypertension. International Journal of Psychiatry in Clinical Practice, 15(3), 214-218. doi: 10.3109/13651501.2011.572168.

Houle, S., Chatterley, T. y Tsuyuki, R. (2014). Multidisciplinary approaches to the management of high blood pressure. Current Opinion in Cardiology, 29(4), 344-353. doi: 10.1097/HCO.0000000000000071.

Instituto Nacional de Salud Pública (2012). Hipertensión arterial en adultos mexicanos: importancia de mejorar el diagnóstico oportuno y el control. Cuernavaca (México): INSP.

Instituto Nacional de Estadística y Geografía (2016). Principales causas de mortalidad por residencia habitual, grupos de edad y sexo del fallecido. México: INEGI. Disponible en línea: http://www.inegi.org.mx/est/contenidos/proyectos/registros/vitales/ mortalidad/tabulados/ConsultaMortalidad.asp.

Jankowska-Polańska, B., Uchmanowicz, I., Dudek, K. y Mazur, G. (2016). Relationship between patients' knowledge and medication adherence among patients with hypertension. Patient Preference and Adherence, 10, 2437-2447. doi: 10.2147/PPA. S117269.

Kubzansky, L. y Kawachi, I. (2000). Going to the heart of the matter: do negative emotions cause coronary heart disease? Journal Psychosomatic Research, 48, 323-337.

López, B., Rodríguez, E., Vázquez, F. y Alcázar, R. (2012). Intervención cognitivo-conductual para el manejo de la ira. Revista Mexicana de Psicología, 29(1), 97-104.

Maatouk, I., Herzog, W., Böhlen, F., Quinzler, R., Löwe, B., Saum, K., Brenner, H. y Wild, B. (2016). Association of hypertension with depression and generalized anxiety symptoms in a large population-based sample of older adults. Journal of Hypertension, 34(9), 1711-1720. doi: 10.1097/HJH.0000000000001006.

Magan, I., Sanz, J., Espinosa, R. y García V., M. (2013). Perfil emocional y cognitivo de la hipertensión arterial esencial mantenida frente a la normotensión. Clínica y Salud, 24, 67-76.

Moscoso, M. y Spielberger, C. (1999). Measuring the experience, expression and control of anger in Latin America: the Spanish Multi-Cultural State-Trait Anger Expression Inventory. Interamerican Journal of Psychology, 332, 29-48.

Organización Mundial de la Salud (2015). Preguntas y respuestas sobre la hipertensión. Ginebra: OMS. Disponible en línea: http://www.who.int/features/qa/82/es/.

Riveros, A., Ceballos, G., Laguna, R. y Sánchez-Sosa, J. (2005). El manejo psicológico de la hipertensión esencial: efectos de una intervención cognitivo-conductual. Revista Latinoamericana de Psicología, 37(3), 493-507.

Riveros, A., Cortazar P., J., Alcázar, F. y Sánchez-Sosa, J. (2005). Efectos de una intervención cognitivo-conductual en la calidad de vida, ansiedad, depresión y condición médica de pacientes diabéticos e hipertensos esenciales. International Journal of Clinical and Health Psychology, 5(3), 445-462.

Riveros, A., Sánchez-Sosa, J. y Del Águila, M. (2009). Inventario de Calidad de Vida y Salud (INCAVISA). México: El Manual Moderno. 
Robles, R., Varela, R., Jurado, S. y Páez, F. (2001). Versión mexicana del Inventario de Ansiedad de Beck: propiedades psicométricas. Revista Mexicana de Psicología, 18, 211-218.

Rodríguez L., M., Varela, M., Rincón H., L., Velasco, M., Caicedo, D., Méndez, F. y Gómez, O. (2015). Prevalencia y factores asociados a la adherencia al tratamiento no farmacológico en pacientes con hipertensión y diabetes en servicios de baja complejidad. Revista de la Facultad Nacional de Salud Pública, 33(2), 192-199.

Ruiz, M., Díaz, M. y Villalobos, A. (2012). Manual de técnicas de modificación de conducta. Bilbao: Descleé de Brouwer.

Siegel, S. y Castellan, J. (1995). Estadística no paramétrica aplicada a las ciencias de la conducta. México: Trillas.

Schoenthaler, A., De la Calle, F., Barrios B., M., Garcia, A., Pitaro, M., Lum, A. y Rosal, M. (2015). A practice-based randomized controlled trial to improve medication adherence among Latinos with hypertension: Study protocol for a randomized controlled trial. Trials, 16, 290-301. doi: 10.1186/s13063-015-0815-x.

Trevisol, D., Moreira, L., Kerkhoff, A., Fuchs, S. y Fuchs, F. (2011). Health-related quality of life and hypertension: A systematic review and meta-analysis of observational studies. Journal of Hypertension, 29(2), 179-188. doi: 10.1097/HJH.0b013e$328340 \mathrm{~d} 76 \mathrm{f}$.

Vermeire, E., Hearnshaw, H., P. Van Royen, P. y Denekens, J. (2001). Patient adherence to treatment: Three decades of research: a comprehensive review. Journal of Clinical Pharmacy and Therapeutics, 26, 331-342.

Xianglong, X., Yunshuang, R., Zumin, S., Lingli, L., Cheng, C. y Yong, Z. (2016). Hypertension impact on health-related quality of life: A cross-sectional survey among middle-aged adults in Chongqing, China. International Journal of Hypertension, Article ID 7404957. doi: 10.1155/2016/7404957.

Vocks, S., Ockenfels, M., Jürgensen, R., Mussgay, L. y Rüddel, H. (2004). Blood pressure reactivity can be reduced by a cognitive behavioral stress management program. International Journal of Behavioral Medicine, 11(2), 63-70. 